Acta Crystallographica Section E

Structure Reports

Online

ISSN 1600-5368

\section{Jonathan C. Burley, ${ }^{a}$ * Jacco van de Streek ${ }^{b}$ and Peter W. Stephens ${ }^{c}$}

${ }^{a}$ University Chemical Laboratory, Lensfield Road, Cambridge CB2 1EW, England,

${ }^{\mathbf{b}}$ Cambridge Crystallographic Data Centre, 12 Union Road, Cambridge CB2 1EZ, England, and ${ }^{\mathrm{C}}$ Department of Physics and Astronomy, SUNY at Stony Brook, Stony Brook, NY 117943800, USA

Correspondence e-mail: jb442@cam.ac.uk

\section{Key indicators}

Powder synchrotron study

$T=290 \mathrm{~K}$

Mean $\sigma(\mathrm{C}-\mathrm{C})=0.006 \AA$

$R$ factor $=0.025$

$w R$ factor $=0.030$

Data-to-parameter ratio $=75.44$

For details of how these key indicators were automatically derived from the article, see http://journals.iucr.org/e.

\title{
Ampicillin trihydrate from synchrotron powder diffraction data
}

The crystal structure of ampicillin trihydrate \{systematic name: $\quad 6-[\mathrm{D}(-)-\alpha$-aminophenylacetamido]penicillanic acid trihydrate\}, $\mathrm{C}_{16} \mathrm{H}_{19} \mathrm{~N}_{3} \mathrm{O}_{4} \mathrm{~S} \cdot 3 \mathrm{H}_{2} \mathrm{O}$, a broad-spectrum $\beta$-lactam antibiotic of the aminopenicillin type, has been determined from synchrotron X-ray powder diffraction data. The three water molecules form an infinite hydrogen-bonded chain through the crystal structure, with hydrogen bonds to the $\mathrm{NH}_{3}^{+}, \mathrm{COO}^{-}, \mathrm{C}=\mathrm{O}$ and $\mathrm{NH}$ groups of the ampicillin molecules.

\section{Comment}

The title compound, (I), has been used as a broad-spectrum antibiotic since 1961. The crystal structure was reported in 1968 (James et al., 1968), but no atomic coordinates were given in the paper or deposited. Boles et al. (1978) published the crystal structure of a related compound, amoxycillin trihydrate. They apparently had access to the atomic coordinates of the crystal structure of compound (I), because in their paper they show that the two crystal structures are isostructural. However, the atomic coordinates of the title compound have not been published to date. We report the crystal structure here, determined from synchrotron X-ray powder diffraction.<smiles>CC1(C)SC2C(NC(=O)C([NH3+])c3ccccc3)C(=O)N2C1C(=O)O</smiles>

The structural model of compound (I) obtained in the present work (Fig. 1a) is both chemically reasonable and in accord with the figures given by James et al. (1968). Selected geometric parameters are given in Table 1 . We note, however, that the hydrogen bond $\mathrm{O} 26^{\prime \prime \prime} \ldots \mathrm{O} 25^{\prime \prime \prime}$ in their Fig. 1, which appears to link four water molecules together into a closed tetramer, is spurious, and instead should have formed a chain (Fig. 1b). Both the pattern of hydrogen bonding, and the positions of the $\mathrm{H}$ atoms of the water molecules in the structure, are chemically sensible and compare well with those from the crystal structure of the isostructural amoxycillin trihydrate (Boles et al., 1978). Details of the $\mathrm{O}-\mathrm{H} \cdots \mathrm{O}$ and $\mathrm{N}-\mathrm{H} \cdots \mathrm{O}$ hydrogen bonds are given in Table 2 and Fig. 1.

\section{Experimental}

The sample of compound (I) was a gift from Setauket Pharmacy, Setauket, New York, USA, in the form of a gelatin capsule of the
Received 7 December 2005 Accepted 12 January 2006 
(a)

(b)
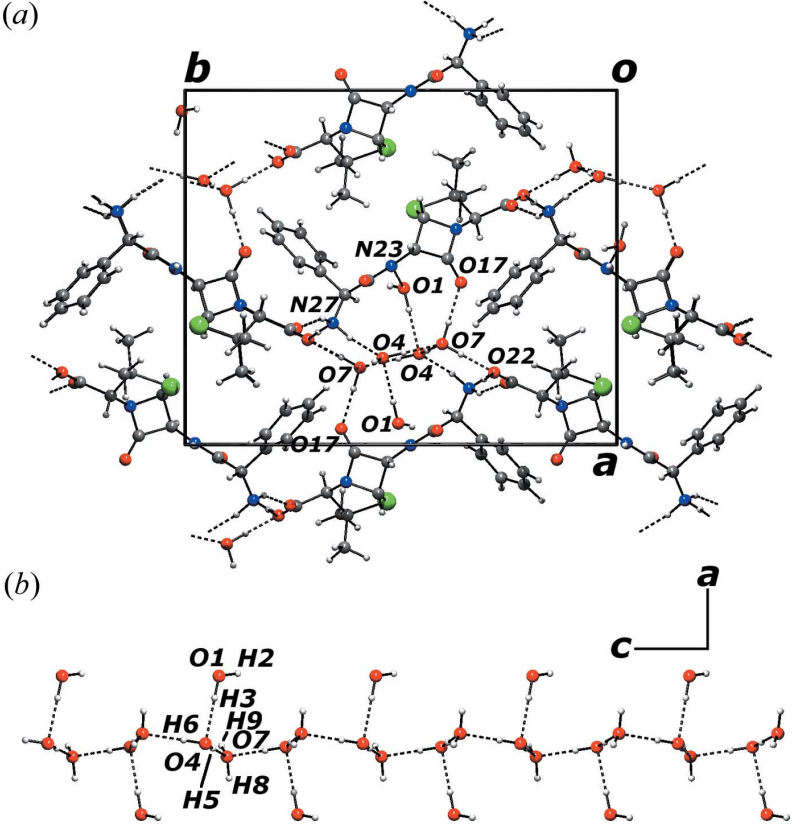

Figure 1

(a) A view, along the $c$ axis, of the crystal structure of compound (I), showing the $\mathrm{O}-\mathrm{H} \cdots \mathrm{O}$ and $\mathrm{N}-\mathrm{H} \cdots \mathrm{O}$ hydrogen bonds as dashed lines (see Table 2 for details). ( $b$ ) A view along the $b$ axis of the hydrogenbonded (dashed lines) water network in the crystal structure of compound (I). [A screw axis is present at $\left(\frac{1}{4}, 0, z\right)$ ].

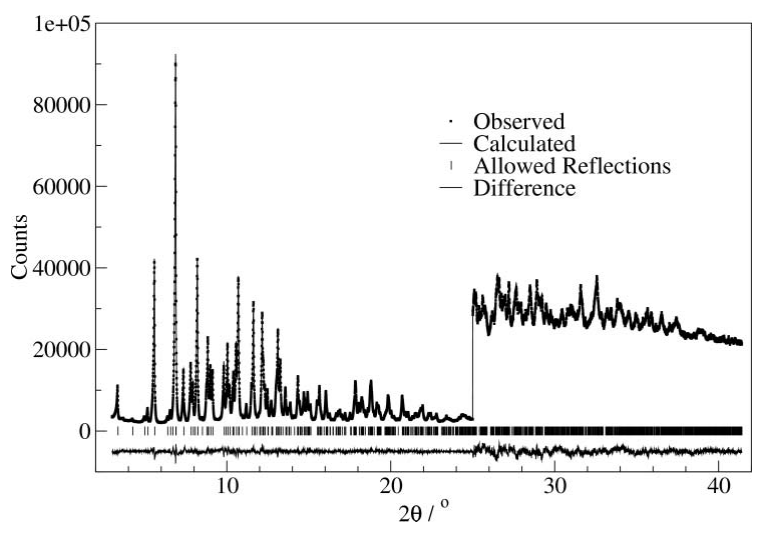

Figure 2

Observed, calculated and difference X-ray powder diffraction profiles for compound (I). The region $25-42^{\circ}$ in $2 \theta$ has been magnified 10 times.

compound. Some of the contents were loaded into a thin-walled glass capillary of $1.5 \mathrm{~mm}$ nominal diameter. Any excipients that might have been present were not crystalline. A diffraction pattern was collected at the X3B1 beamline of the National Synchrotron Light Source, Brookhaven National Laboratory. The wavelength of 0.7003 (1) $\AA$ was selected by a double $\mathrm{Si}(111)$ monochromator and the diffracted beam analyzed by a $\mathrm{Ge}(111)$ crystal before the detector. The beam on the sample had dimensions $2 \mathrm{~mm} \times 8 \mathrm{~mm}$. Data were collected from $2 \theta=3-41.6^{\circ}$ in steps of $0.005^{\circ}$, with counting time increasing quadratically from $1-8 \mathrm{~s}$ per point. The incident beam was monitored by an ion chamber, which was used to normalize the data for decay and fluctuations of the intensity.
Crystal data

$\mathrm{C}_{16} \mathrm{H}_{19} \mathrm{~N}_{3} \mathrm{O}_{4} \mathrm{~S} \cdot 3 \mathrm{H}_{2} \mathrm{O}$

$M_{r}=403.06$

Orthorhombic, $P 2_{1} 2_{1} 2_{1}$

$a=15.52275(16) \AA$

$b=18.9256(3) \AA$

$c=6.67375(8) \AA$

$V=1960.60(3) \AA^{3}$

$Z=4$

\section{Data collection}

Huber 424 Q -2Q diffractometer on X3B1 beamline

Specimen mounting: Lindemann glass capillary

Specimen mounted in transmission mode

\section{Refinement}

$R_{p}=0.025$

$R_{\text {wp }}=0.030$

$R_{\text {exp }}=0.013$

$S=2.37$

$2 \theta_{\min }=3,2 \theta_{\max }=41.6^{\circ}$

Increment in $2 \theta=0.005^{\circ}$

Wavelength of incident radiation: $0.7003 \AA$

Excluded region(s): none

Profile function: $\mathrm{CW}$ profile function number 3 with 19 terms. Pseudo-Voigt profile coefficients as parameterized in Thompson $e t$
$D_{x}=1.367 \mathrm{Mg} \mathrm{m}^{-3}$

Synchrotron radiation

$\lambda=0.7003 \AA$

$T=290 \mathrm{~K}$

Specimen shape: cylinder

$0.7 \times 20 \mathrm{~mm}$

Particle morphology: powder, white

Scan method: step

$T=290 \mathrm{~K}$

$2 \theta_{\min }=3,2 \theta_{\max }=41.6^{\circ}$

Increment in $2 \theta=0.005^{\circ}$

al. (1987). Asymmetry correction of Finger et al. (1994). Peak tails are ignored where the intensity is below 0.0010 times the peak 10788 reflections

143 parameters

$\mathrm{H}$-atom parameters constrained

Weighting scheme based on

measured s.u. values

$(\Delta / \sigma)_{\max }=0.03$

Preferred orientation correction: none
Table 1

Selected geometric parameters $\left(\AA{ }^{\circ}\right)$.

\begin{tabular}{lrlr}
\hline S10-C11 & $1.859(4)$ & $\mathrm{N} 13-\mathrm{C} 12$ & $1.473(5)$ \\
S10-C14 & $1.797(3)$ & $\mathrm{N} 13-\mathrm{C} 14$ & $1.506(5)$ \\
O17-C16 & $1.201(5)$ & $\mathrm{N} 13-\mathrm{C} 16$ & $1.384(5)$ \\
O21-C20 & $1.222(6)$ & $\mathrm{N} 23-\mathrm{C} 15$ & $1.433(5)$ \\
$\mathrm{O} 22-\mathrm{C} 20$ & $1.273(6)$ & $\mathrm{N} 23-\mathrm{C} 24$ & $1.348(7)$ \\
$\mathrm{O} 25-\mathrm{C} 24$ & $1.213(5)$ & $\mathrm{N} 27-\mathrm{C} 26$ & $1.475(6)$ \\
& & & \\
C11-S10-C14 & $90.10(18)$ & $\mathrm{N} 23-\mathrm{C} 15-\mathrm{C} 16$ & $115.5(3)$ \\
$\mathrm{C} 12-\mathrm{N} 13-\mathrm{C} 14$ & $117.0(3)$ & $\mathrm{N} 23-\mathrm{C} 15-\mathrm{C} 14$ & $116.5(3)$ \\
$\mathrm{C} 12-\mathrm{N} 13-\mathrm{C} 16$ & $128.1(3)$ & $\mathrm{O} 17-\mathrm{C} 16-\mathrm{C} 15$ & $135.4(3)$ \\
$\mathrm{C} 14-\mathrm{N} 13-\mathrm{C} 16$ & $93.1(3)$ & $\mathrm{O} 17-\mathrm{C} 16-\mathrm{N} 13$ & $131.0(4)$ \\
$\mathrm{C} 15-\mathrm{N} 23-\mathrm{C} 24$ & $123.3(3)$ & $\mathrm{N} 13-\mathrm{C} 16-\mathrm{C} 15$ & $93.0(3)$ \\
S10-C11-C19 & $107.5(3)$ & $\mathrm{O} 21-\mathrm{C} 20-\mathrm{C} 12$ & $118.0(4)$ \\
S10-C11-C12 & $104.6(2)$ & $\mathrm{O} 21-\mathrm{C} 20-\mathrm{O} 22$ & $126.3(4)$ \\
S10-C11-C18 & $110.0(3)$ & $\mathrm{O} 22-\mathrm{C} 20-\mathrm{C} 12$ & $115.6(4)$ \\
$\mathrm{N} 13-\mathrm{C} 12-\mathrm{C} 11$ & $105.6(3)$ & $\mathrm{O} 25-\mathrm{C} 24-\mathrm{N} 23$ & $124.5(5)$ \\
$\mathrm{N} 13-\mathrm{C} 12-\mathrm{C} 20$ & $112.8(3)$ & $\mathrm{O} 25-\mathrm{C} 24-\mathrm{C} 26$ & $122.1(5)$ \\
$\mathrm{N} 13-\mathrm{C} 14-\mathrm{C} 15$ & $87.1(2)$ & $\mathrm{N} 23-\mathrm{C} 24-\mathrm{C} 26$ & $113.2(3)$ \\
S10-C14-N13 & $103.6(2)$ & $\mathrm{N} 27-\mathrm{C} 26-\mathrm{C} 24$ & $110.0(3)$ \\
S10-C14-C15 & $119.5(2)$ & $\mathrm{N} 27-\mathrm{C} 26-\mathrm{C} 28$ & $112.9(3)$ \\
\hline
\end{tabular}

Table 2

Hydrogen-bond geometry $\left(\AA{ }^{\circ}\right)$.

\begin{tabular}{|c|c|c|c|c|}
\hline$D-\mathrm{H} \cdots A$ & $D-\mathrm{H}$ & $\mathrm{H} \cdots A$ & $D \cdots A$ & $D-\mathrm{H} \cdots A$ \\
\hline $\mathrm{O} 1-\mathrm{H} 3 \cdots \mathrm{O} 4$ & 0.98 & 2.02 & 2.9968 & 180 \\
\hline $\mathrm{O} 4-\mathrm{H} 5 \cdots \mathrm{O} 7^{\mathrm{i}}$ & 0.98 & 1.80 & 2.7850 & 180 \\
\hline $\mathrm{O} 4-\mathrm{H} 6 \cdots \mathrm{O} 7$ & 0.98 & 1.82 & 2.7967 & 180 \\
\hline $\mathrm{O} 7-\mathrm{H} 8 \cdots \mathrm{O} 17$ & 0.98 & 1.84 & 2.8225 & 180 \\
\hline $\mathrm{O} 7-\mathrm{H} 9 \cdots \mathrm{O} 22^{2 \mathrm{ii}}$ & 0.98 & 1.74 & 2.7166 & 179 \\
\hline $\mathrm{N} 27-\mathrm{H} 38 \cdots \mathrm{O} 4^{\mathrm{iii}}$ & $1.001(15)$ & 1.86 & 2.8272 & 161 \\
\hline $\mathrm{N} 27-\mathrm{H} 39 \cdots \mathrm{O} 21^{\mathrm{iv}}$ & $1.00(3)$ & $1.83(3)$ & $2.742(6)$ & $151.1(15)$ \\
\hline
\end{tabular}




\begin{tabular}{lllll}
$D-\mathrm{H} \cdots A$ & $D-\mathrm{H}$ & $\mathrm{H} \cdots A$ & $D \cdots A$ & $D-\mathrm{H} \cdots A$ \\
\hline $\mathrm{N} 27-\mathrm{H} 40 \cdots \mathrm{O} 22^{\mathrm{v}}$ & $1.00(3)$ & $1.80(2)$ & $2.688(6)$ & $147(2)$ \\
$\mathrm{N} 23-\mathrm{H} 51 \cdots \mathrm{O} 1$ & $1.002(10)$ & 1.97 & 2.9161 & 156 \\
\hline
\end{tabular}

Symmetry codes: (i) $\quad-x+\frac{3}{2},-y+1, z-\frac{1}{2}$; $\quad$ (ii) $\quad x+\frac{1}{2},-y+\frac{1}{2},-z+1$; $\quad$ (iii) $-x+\frac{3}{2},-y+1, z+\frac{1}{2}$; (iv) $-x+1, y+\frac{1}{2},-z+\frac{3}{2} ;$ (v) $-x+1, y+\frac{1}{2},-z+\frac{1}{2}$.

The starting model for Rietveld refinement was obtained by solving the crystal structure from the powder diffraction pattern. This also provided an independent check that the published crystal structure is correct. However, with the crystal structure being known, its determination from the powder pattern is mainly academic. The crystal structure was determined with the program $D A S H$ (David et al., 2004). For the structure solution, the data were truncated at $22.855^{\circ}$ in $2 \theta$, corresponding to a real-space resolution of $1.767 \AA$. The background was subtracted with a Bayesian high-pass filter (David \& Sivia, 2001). Peak positions for indexing were obtained by fitting with an asymmetry-corrected Voigt function, followed by indexing with the program DICVOL (Boultif \& Louer, 1991). An orthorhombic and several monoclinic unit cells were obtained. However, all the monoclinic unit cells were pseudo-orthorhombic with nearly the same parameters as the orthorhombic cell, indicating that the orthorhombic unit cell is the correct one. The figures of merit given by $D I C V O L$ were $M(20)=62.1$ and $F(20)=337.1(0.0014,42)$. The space group reported for the single-crystal structure, $P 2_{1} 2_{1} 2_{1}$, gave an excellent Pawley fit.

Simulated annealing was used to solve the crystal structure of compound (I) from the powder pattern in direct space. The starting molecular geometry was taken from the anhydrate (Boles \& Girven, 1976), entry AMCILL in the Cambridge Structural Database (Allen, 2002). The molecule is a zwitterion, in agreement with the singlecrystal study. Because $\mathrm{H}$ atoms do not contribute significantly to the powder diffraction pattern, due to their low X-ray scattering power, they were ignored during the structure solution process. Hence, the water molecule can be reduced to an $\mathrm{O}$ atom, which reduces its number of degrees of freedom from six to three. The molecule has five flexible torsion angles, which, when combined with the three water molecules, give a total of 20 degrees of freedom. In ten simulated annealing runs, the correct crystal structure was found twice, with a profile $\chi^{2}=81.7,11$ times the Pawley $\chi^{2}$. The next-best crystal structure had a profile $\chi^{2}=240$. The low success rate and high profile $\chi^{2}$ are caused by the high $R$ factor of $10.6 \%$ of the crystal structure of AMCILL from which the starting model was taken; when the structure solution was repeated with a better starting model (obtained from Rietveld refinement against the powder data), the correct structure was found four times in ten runs, with a profile $\chi^{2}=20$, less than three times the Pawley $\chi^{2}$.

The background subtraction, peak fitting, indexing, Pawley refinement and simulated-annealing algorithms used are as implemented in the program $D A S H$.

For the Rietveld refinement (Fig. 2), $\mathrm{H}$ atoms were included in the initial model in calculated positions. Bond lengths, bond angles and planar groupings were subjected to suitable constraints, including bonds to $\mathrm{H}$ atoms. Data were included to $41.42^{\circ}$ in $2 \theta$, corresponding to a real-space resolution of $0.99 \AA$. The refinement was not particularly sensitive to the position of the water $\mathrm{H}$ atoms and these were included in calculated positions, with the water molecules being fixed in position for the final refinement cycles. The refinement proceeded smoothly to reach a minimum characterized by an excellent fit to the diffraction profile $\left(\chi^{2}=5.637, R_{\mathrm{p}}=0.0296, R_{\mathrm{wp}}=0.0296\right.$ and $R_{\mathrm{Bragg}}=$ 0.0295).

Data collection: local software; cell refinement: GSAS (Larson \& Von Dreele, 2000); data reduction: local software; program(s) used to solve structure: DASH (David et al., 2004); program(s) used to refine structure: GSAS; molecular graphics: PLATON (Spek, 2003); software used to prepare material for publication: PLATON.

JB thanks Jesus College, Cambridge, for the award of a Junior Research Fellowship. Use of the National Synchrotron Light Source, Brookhaven National Laboratory, was supported by the US Department of Energy, Office of Science, Office of Basic Energy Sciences, under Contract No. DeAC02-98CH10886.

\section{References}

Allen, F. H. (2002). Acta Cryst. B58, 380-388.

Boles, M. O. \& Girven, R. J. (1976). Acta Cryst. B32, 2279-2284.

Boles, M. O., Girven, R. J. \& Gane, P. A. C. (1978). Acta Cryst. B34, 461-466.

Boultif, A. \& Louer, D. (1991). J. Appl. Cryst. 24, 987-993.

David, W. I. F., Shankland, K., Van de Streek, J., Pidcock, E. \& Motherwell, S. (2004). DASH. Version 3.0. Cambridge Crystallographic Data Centre, 12 Union Road, Cambridge, England..

David, W. I. F. \& Sivia, D. S. (2001). J. Appl. Cryst. 34, 318-324.

Finger, L. W., Cox, D. E. \& Jephcoat, A. P. (1994). J. Appl. Cryst. 27, 892-900. James, M. N. G., Hall, D. \& Hodgkin, D. C. (1968). Nature, 220, 168-170.

Larson, A. C. \& Von Dreele, R. B. (2000). GSAS. Report LAUR 86-748. Los Alamos National Laboratory, New Mexico, USA.

Spek, A. L. (2003). J. Appl. Cryst. 36, 7-13.

Thompson, P., Cox, D. E. \& Hastings, J. B. (1987). J. Appl. Cryst. 20,79-83. 


\title{
supporting information
}

Acta Cryst. (2006). E62, o797-o799 [https://doi.org/10.1107/S1600536806001371]

\section{Ampicillin trihydrate from synchrotron powder diffraction data}

\author{
Jonathan C. Burley, Jacco van de Streek and Peter W. Stephens
}

6-[D(-)- $\alpha$-Aminophenylacetamido]penicillanic acid trihydrate

Crystal data

$\mathrm{C}_{16} \mathrm{H}_{19} \mathrm{~N}_{3} \mathrm{O}_{4} \mathrm{~S} \cdot 3 \mathrm{H}_{2} \mathrm{O}$

$M_{r}=403.06$

Orthorhombic, $P 2_{1} 2_{1} 2_{1}$

$a=15.52275(16) \AA$

$b=18.9256(3) \AA$

$c=6.67375(8) \AA$

$V=1960.60(3) \AA^{3}$

\section{Data collection}

Diffractometer $\mathrm{x} 3 \mathrm{~b} 1$

Radiation source: Brookhaven NSLS

Specimen mounting: Lindemann glass capillary

\section{Refinement}

Least-squares matrix: full

$R_{\mathrm{p}}=0.025$

$R_{\text {wp }}=0.030$

$R_{\text {exp }}=0.013$

$R\left(F^{2}\right)=0.02950$

Excluded region(s): none
$Z=4$

$D_{\mathrm{x}}=1.367 \mathrm{Mg} \mathrm{m}^{-3}$

Synchrotron radiation, $\lambda=0.7003 \AA$

$T=290 \mathrm{~K}$

Particle morphology: powder

white

cylinder, $0.7 \times 20 \mathrm{~mm}$

Data collection mode: transmission

Scan method: step

Profile function: $\mathrm{CW}$ Profile function number 3 with 19 terms Pseudovoigt profile coefficients as parameterized in Thompson et al. (1987).

Asymmetry correction of Finger et al. (1994).

$\# 1(\mathrm{GU})=0.000 \# 2(\mathrm{GV})=0.000 \# 3(\mathrm{GW})=$ $0.000 \# 4(\mathrm{GP})=0.000 \# 5(\mathrm{LX})=2.213 \# 6(\mathrm{LY})=$ $33.715 \# 7(\mathrm{~S} / \mathrm{L})=0.0130 \# 8(\mathrm{H} / \mathrm{L})=0.0130$ $\# 9($ trns $)=0.00 \# 10(\mathrm{shft})=0.0832 \# 11(\mathrm{stec})=$ $0.00 \# 12(\mathrm{ptec})=0.00 \# 13(\mathrm{sfec})=0.00 \# 14(\mathrm{~L} 11)$ $=0.000 \# 15(\mathrm{~L} 22)=0.000 \# 16(\mathrm{~L} 33)=0.000$ $\# 17(\mathrm{~L} 12)=0.000 \# 18(\mathrm{~L} 13)=0.000 \# 19(\mathrm{~L} 23)=$ 0.000 Peak tails are ignored where the intensity is below 0.0010 times the peak Aniso. broadening axis 0.00 .01 .0

143 parameters 136 restraints

$\mathrm{H}$-atom parameters constrained

Weighting scheme based on measured s.u.'s $(\Delta / \sigma)_{\max }=0.03$

Background function: GSAS Background function number 1 with 9 terms. Shifted Chebyshev function of 1st kind 1: 2261.502 : -239.085 3: 47.0827 4: -155.949 5: 139.395 6: -237.334 7: 231.040 8: -74.9779 9: 19.4239 10: $-0.402815$ 


\section{Special details}

Geometry. Bond distances, angles etc. have been calculated using the rounded fractional coordinates. All su's are estimated from the variances of the (full) variance-covariance matrix. The cell e.s.d.'s are taken into account in the estimation of distances, angles and torsion angles

Fractional atomic coordinates and isotropic or equivalent isotropic displacement parameters $\left(\AA^{2}\right)$

\begin{tabular}{|c|c|c|c|c|}
\hline & $x$ & $y$ & $z$ & $U_{\text {iso }} * / U_{\text {eq }}$ \\
\hline S10 & $0.33689(14)$ & $0.46707(11)$ & 0.2754 & $0.0350(4)^{*}$ \\
\hline $\mathrm{O} 17$ & $0.5429(2)$ & $0.3613(2)$ & $0.5525(7)$ & $0.0350(4)^{*}$ \\
\hline $\mathrm{O} 21$ & 0.3263 & $0.2430(2)$ & $0.6113(6)$ & $0.0350(4)^{*}$ \\
\hline $\mathrm{O} 22$ & $0.2965(3)$ & $0.2156(2)$ & $0.2943(6)$ & $0.0350(4)^{*}$ \\
\hline $\mathrm{O} 25$ & $0.5385(3)$ & $0.5837(2)$ & $0.7961(5)$ & $0.0350(4)^{*}$ \\
\hline N13 & $0.3923(2)$ & $0.37247(18)$ & $0.5272(5)$ & $0.0350(4)^{*}$ \\
\hline $\mathrm{N} 23$ & $0.4964(3)$ & $0.5225(2)$ & $0.5209(5)$ & $0.0350(4)^{*}$ \\
\hline $\mathrm{N} 27$ & $0.6573(3)$ & $0.6581(2)$ & $0.5579(6)$ & $0.0350(4)^{*}$ \\
\hline C11 & $0.2977(2)$ & $0.37521(15)$ & $0.2374(5)$ & $0.0350(4)^{*}$ \\
\hline $\mathrm{C} 12$ & $0.3623(2)$ & $0.32784(17)$ & $0.3604(6)$ & $0.0350(4)^{*}$ \\
\hline C14 & $0.3598(2)$ & $0.4474(2)$ & $0.5333(4)$ & $0.0350(4)^{*}$ \\
\hline C15 & $0.4496(2)$ & $0.46826(18)$ & $0.6238(5)$ & $0.0350(4)^{*}$ \\
\hline C16 & $0.4759(2)$ & $0.39222(19)$ & $0.5728(8)$ & $0.0350(4)^{*}$ \\
\hline $\mathrm{C} 18$ & $0.2064(3)$ & $0.3683(3)$ & $0.3136(8)$ & $0.0350(4)^{*}$ \\
\hline C19 & 0.3001 & $0.3604(3)$ & $0.0177(6)$ & $0.0350(4)^{*}$ \\
\hline $\mathrm{C} 20$ & $0.3239(4)$ & $0.2563(2)$ & $0.4322(6)$ & $0.0350(4)^{*}$ \\
\hline $\mathrm{C} 24$ & $0.5369(4)$ & $0.5759(3)$ & $0.6158(5)$ & $0.0350(4)^{*}$ \\
\hline $\mathrm{C} 26$ & $0.5768(2)$ & $0.6298(2)$ & $0.4722(5)$ & $0.0350(4)^{*}$ \\
\hline $\mathrm{C} 28$ & $0.5114(3)$ & $0.6874(3)$ & $0.4222(6)$ & $0.0350(4)^{*}$ \\
\hline $\mathrm{C} 29$ & $0.4704(4)$ & $0.6867(3)$ & $0.2375(7)$ & $0.0350(4)^{*}$ \\
\hline $\mathrm{C} 30$ & 0.4035 & 0.7333 & $0.2002(7)$ & $0.0350(4)^{*}$ \\
\hline C31 & $0.3851(4)$ & $0.7862(3)$ & $0.3350(8)$ & $0.0350(4)^{*}$ \\
\hline $\mathrm{C} 32$ & 0.4208 & 0.7839 & $0.5249(7)$ & $0.0350(4)^{*}$ \\
\hline $\mathrm{C} 33$ & $0.4853(4)$ & 0.7355 & $0.5675(6)$ & $0.0350(4)^{*}$ \\
\hline $\mathrm{O} 1$ & 0.55980 & 0.49350 & 0.11790 & $0.0350(4)^{*}$ \\
\hline $\mathrm{O} 4$ & 0.74455 & 0.45750 & 0.19890 & $0.0350(4)^{*}$ \\
\hline $\mathrm{O} 7$ & 0.71633 & 0.40465 & 0.58470 & $0.0350(4)^{*}$ \\
\hline H34 & $0.4160(4)$ & 0.3174 & $0.2832(9)$ & $0.0350(4)^{*}$ \\
\hline H35 & 0.3068 & $0.4564(4)$ & $0.6142(8)$ & $0.0350(4)^{*}$ \\
\hline H36 & $0.4413(5)$ & $0.4787(4)$ & $0.7695(7)$ & $0.0350(4) *$ \\
\hline H37 & $0.5926(5)$ & $0.6037(4)$ & $0.3330(8)$ & $0.0350(4)^{*}$ \\
\hline H38 & $0.6990(11)$ & $0.6186(5)$ & $0.581(6)$ & $0.0350(4)^{*}$ \\
\hline H39 & $0.6446(7)$ & $0.682(2)$ & $0.688(3)$ & $0.0350(4)^{*}$ \\
\hline $\mathrm{H} 40$ & $0.6835(16)$ & $0.6927(18)$ & $0.463(3)$ & $0.0350(4)^{*}$ \\
\hline H41 & $0.4940(15)$ & $0.6530(13)$ & $0.1193(16)$ & $0.0350(4)^{*}$ \\
\hline H42 & $0.3735(13)$ & $0.7338(9)$ & $0.0544(16)$ & $0.0350(4)^{*}$ \\
\hline H43 & $0.3981(15)$ & $0.8194(12)$ & $0.6399(17)$ & $0.0350(4)^{*}$ \\
\hline H44 & $0.5112(14)$ & $0.7321(11)$ & $0.7174(14)$ & $0.0350(4)^{*}$ \\
\hline $\mathrm{H} 45$ & $0.1765(10)$ & $0.4198(5)$ & $0.317(6)$ & $0.0350(4)^{*}$ \\
\hline H46 & $0.1705(9)$ & $0.3342(18)$ & $0.214(4)$ & $0.0350(4)^{*}$ \\
\hline
\end{tabular}




\begin{tabular}{lllll}
$\mathrm{H} 47$ & $0.2068(5)$ & $0.346(2)$ & $0.463(3)$ & $0.0350(4)^{*}$ \\
$\mathrm{H} 48$ & $0.3658(5)$ & $0.364(2)$ & $-0.0353(16)$ & $0.0350(4)^{*}$ \\
$\mathrm{H} 49$ & $0.275(2)$ & $0.3082(8)$ & $-0.0102(13)$ & $0.0350(4)^{*}$ \\
$\mathrm{H} 50$ & $0.261(2)$ & $0.3986(13)$ & $-0.0597(12)$ & $0.0350(4)^{*}$ \\
$\mathrm{H} 51$ & $0.503(3)$ & $0.5188(16)$ & $0.3719(11)$ & $0.0350(4)^{*}$ \\
$\mathrm{H} 52$ & $0.3394(16)$ & $0.8272(10)$ & $0.297(2)$ & $0.0350(4)^{*}$ \\
$\mathrm{H} 2$ & 0.55279 & 0.52556 & 1.00373 & $0.0350(4)^{*}$ \\
$\mathrm{H} 3$ & 0.62022 & 0.48173 & 0.14439 & $0.0350(4)^{*}$ \\
$\mathrm{H} 5$ & 0.75832 & 0.50601 & 0.15871 & $0.0350(4)^{*}$ \\
$\mathrm{H} 6$ & 0.73466 & 0.43898 & 0.33409 & $0.0350(4)^{*}$ \\
$\mathrm{H} 8$ & 0.65604 & 0.38978 & 0.57372 & $0.0350(4)^{*}$ \\
H9 & 0.74556 & 0.36129 & 0.62735 & $0.0350(4)^{*}$ \\
\hline
\end{tabular}

Geometric parameters $\left(\AA,^{\circ}\right)$

\begin{tabular}{|c|c|c|c|}
\hline $\mathrm{S} 10-\mathrm{C} 11$ & 1.859 (4) & $\mathrm{C} 14-\mathrm{C} 15$ & $1.570(4)$ \\
\hline $\mathrm{S} 10-\mathrm{C} 14$ & $1.797(3)$ & $\mathrm{C} 15-\mathrm{C} 16$ & $1.534(5)$ \\
\hline $\mathrm{O} 17-\mathrm{C} 16$ & $1.201(5)$ & $\mathrm{C} 24-\mathrm{C} 26$ & $1.531(6)$ \\
\hline $\mathrm{O} 21-\mathrm{C} 20$ & $1.222(6)$ & $\mathrm{C} 26-\mathrm{C} 28$ & $1.527(6)$ \\
\hline $\mathrm{O} 22-\mathrm{C} 20$ & $1.273(6)$ & $\mathrm{C} 28-\mathrm{C} 29$ & $1.387(7)$ \\
\hline $\mathrm{O} 25-\mathrm{C} 24$ & $1.213(5)$ & $\mathrm{C} 28-\mathrm{C} 33$ & $1.390(7)$ \\
\hline $\mathrm{O} 1-\mathrm{H} 3$ & 0.9800 & $\mathrm{C} 29-\mathrm{C} 30$ & $1.385(8)$ \\
\hline $\mathrm{O} 1-\mathrm{H} 2^{\mathrm{i}}$ & 0.9800 & $\mathrm{C} 30-\mathrm{C} 31$ & $1.376(8)$ \\
\hline $\mathrm{O} 4-\mathrm{H} 5$ & 0.9800 & $\mathrm{C} 31-\mathrm{C} 32$ & $1.384(7)$ \\
\hline $\mathrm{O} 4-\mathrm{H} 6$ & 0.9800 & $\mathrm{C} 32-\mathrm{C} 33$ & $1.387(8)$ \\
\hline $\mathrm{O} 7-\mathrm{H} 9$ & 0.9800 & $\mathrm{C} 12-\mathrm{H} 34$ & $1.000(7)$ \\
\hline $\mathrm{O} 7-\mathrm{H} 8$ & 0.9800 & C14-H35 & $0.999(6)$ \\
\hline $\mathrm{N} 13-\mathrm{C} 12$ & $1.473(5)$ & C15-H36 & $1.001(6)$ \\
\hline $\mathrm{N} 13-\mathrm{C} 14$ & $1.506(5)$ & $\mathrm{C} 18-\mathrm{H} 47$ & $1.08(2)$ \\
\hline $\mathrm{N} 13-\mathrm{C} 16$ & $1.384(5)$ & $\mathrm{C} 18-\mathrm{H} 45$ & $1.080(12)$ \\
\hline $\mathrm{N} 23-\mathrm{C} 15$ & $1.433(5)$ & $\mathrm{C} 18-\mathrm{H} 46$ & $1.08(3)$ \\
\hline $\mathrm{N} 23-\mathrm{C} 24$ & $1.348(7)$ & C19-H49 & $1.078(18)$ \\
\hline $\mathrm{N} 27-\mathrm{C} 26$ & $1.475(6)$ & $\mathrm{C} 19-\mathrm{H} 48$ & $1.082(10)$ \\
\hline N23-H51 & $1.002(10)$ & C19- & $1.08(2)$ \\
\hline N27-H39 & $1.00(3)$ & C26- & $1.080(7)$ \\
\hline $\mathrm{N} 27-\mathrm{H} 38$ & $1.001(15)$ & $\mathrm{C} 29-\mathrm{H} 41$ & 1.079 (19) \\
\hline $\mathrm{N} 27-\mathrm{H} 40$ & $1.00(3)$ & C30- $\mathrm{H} 42$ & $1.079(14)$ \\
\hline $\mathrm{C} 11-\mathrm{C} 18$ & $1.511(6)$ & C31-H52 & $1.08(2)$ \\
\hline $\mathrm{C} 11-\mathrm{C} 12$ & $1.576(5)$ & $\mathrm{C} 32-\mathrm{H} 43$ & 1.079 (19) \\
\hline $\mathrm{C} 11-\mathrm{C} 19$ & $1.493(5)$ & C33-H44 & $1.080(13)$ \\
\hline $\mathrm{C} 12-\mathrm{C} 20$ & $1.555(5)$ & & \\
\hline $\mathrm{S} 10 \cdots \mathrm{N} 13$ & $2.602(4)$ & $\mathrm{H} 2 \cdots \mathrm{H} 36$ & 2.5000 \\
\hline $\mathrm{S} 10 \cdots \mathrm{N} 23$ & $3.149(5)$ & $\mathrm{H} 2 \cdots \mathrm{H} 51^{\mathrm{x}}$ & 2.5800 \\
\hline 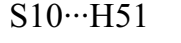 & $2.83(4)$ & $\mathrm{H} 2 \cdots \mathrm{H} 3^{\mathrm{x}}$ & 1.6300 \\
\hline $\mathrm{S} 10 \cdots \mathrm{H} 35^{\mathrm{ii}}$ & $2.869(6)$ & $\mathrm{H} 2 \cdots \mathrm{O} 25$ & 1.7800 \\
\hline $\mathrm{S} 10 \cdots \mathrm{H} 50^{\mathrm{iii}}$ & $3.16(2)$ & $\mathrm{H} 2 \cdots \mathrm{C} 24$ & 2.7700 \\
\hline $\mathrm{O} 1 \cdots \mathrm{O} 4$ & 2.9968 & $\mathrm{H} 3 \cdots \mathrm{O} 4$ & 2.0200 \\
\hline
\end{tabular}




\begin{tabular}{|c|c|c|c|}
\hline $\mathrm{O} 1 \cdots \mathrm{O} 25^{\mathrm{i}}$ & 2.7633 & H3 $\cdots \mathrm{H} 51$ & 2.4700 \\
\hline $\mathrm{O} 1 \cdots \mathrm{N} 23$ & 2.9161 & $\mathrm{H} 3 \cdots \mathrm{H} 5$ & 2.1900 \\
\hline $\mathrm{O} 4 \cdots \mathrm{N} 27^{\mathrm{iv}}$ & 2.8272 & H3 $\cdots \mathrm{H} 6$ & 2.3300 \\
\hline $\mathrm{O} 4 \cdots \mathrm{O}^{\text {iv }}$ & 2.7850 & $\mathrm{H} 5 \cdots \mathrm{O} 7^{\mathrm{iv}}$ & 1.8000 \\
\hline $\mathrm{O} 4 \cdots \mathrm{O} 1$ & 2.9968 & $\mathrm{H} 5 \cdots \mathrm{H} 6^{\mathrm{iv}}$ & 2.4100 \\
\hline $\mathrm{O} 4 \cdots \mathrm{O} 7$ & 2.7967 & $\mathrm{H} 5 \cdots \mathrm{H} 9^{\text {iv }}$ & 2.5200 \\
\hline $\mathrm{O} 7 \cdots \mathrm{O} 17$ & 2.8225 & $\mathrm{H} 5 \cdots \mathrm{H} 38^{\mathrm{iv}}$ & 2.5000 \\
\hline $\mathrm{O} 7 \cdots \mathrm{O} 4$ & 2.7967 & H5 $\cdots \mathrm{H} 3$ & 2.1900 \\
\hline $\mathrm{O} 7 \cdots \mathrm{O} 4^{\mathrm{v}}$ & 2.7850 & $\mathrm{H} 5 \cdots \mathrm{H} 8^{\text {iv }}$ & 2.4500 \\
\hline $\mathrm{O} 7 \cdots \mathrm{O} 22^{\mathrm{vi}}$ & 2.7166 & $\mathrm{H} 6 \cdots \mathrm{O} 7$ & 1.8200 \\
\hline $\mathrm{O} 17 \cdots \mathrm{N} 23$ & $3.142(5)$ & $\mathrm{H} 6 \cdots \mathrm{H} 3$ & 2.3300 \\
\hline $\mathrm{O} 17 \cdots \mathrm{C} 31^{\mathrm{vii}}$ & $3.156(7)$ & H6 $\cdots$ H9 & 2.4500 \\
\hline $\mathrm{O} 17 \cdots \mathrm{C} 30^{\mathrm{vii}}$ & $3.067(7)$ & $\mathrm{H} 6 \cdots{ }^{\prime} 5^{v}$ & 2.4100 \\
\hline $\mathrm{O} 17 \cdots \mathrm{O} 7$ & 2.8225 & H6 $\cdots$ H8 & 2.2200 \\
\hline $\mathrm{O} 17 \cdots \mathrm{C} 32^{\mathrm{viii}}$ & $3.228(7)$ & $\mathrm{H} 6 \cdots \mathrm{H} 38^{\text {iv }}$ & 2.2600 \\
\hline $\mathrm{O} 21 \cdots \mathrm{N} 13$ & $2.715(5)$ & $\mathrm{H} 6 \cdots \mathrm{H} 52^{\mathrm{vii}}$ & 2.5600 \\
\hline $\mathrm{O} 21 \cdots \mathrm{N} 27^{\mathrm{viii}}$ & $2.742(6)$ & $\mathrm{H} 8 \cdots \mathrm{O} 17$ & 1.8400 \\
\hline $\mathrm{O} 22 \cdots \mathrm{C} 26^{\mathrm{vii}}$ & $3.109(5)$ & $\mathrm{H} 8 \cdots \mathrm{H} 43^{\text {viii }}$ & 2.4800 \\
\hline $\mathrm{O} 22 \cdots \mathrm{O} 7^{\mathrm{ix}}$ & 2.7166 & $\mathrm{H} 8 \cdots \mathrm{C} 16$ & 2.8000 \\
\hline $\mathrm{O} 22 \cdots \mathrm{C} 19$ & $3.305(6)$ & 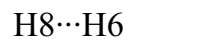 & 2.2200 \\
\hline $\mathrm{O} 22 \cdots \mathrm{C} 28^{\mathrm{vii}}$ & $3.356(6)$ & $\mathrm{H} 8 \cdots \mathrm{H} 5^{\mathrm{v}}$ & 2.4500 \\
\hline $\mathrm{O} 22 \cdots \mathrm{C} 18$ & $3.213(7)$ & H9 $\cdots \mathrm{H} 6$ & 2.4500 \\
\hline $\mathrm{O} 22 \cdots \mathrm{N} 27^{\mathrm{vii}}$ & $2.688(6)$ & $\mathrm{H} 9 \cdots \mathrm{H} 5^{\mathrm{v}}$ & 2.5200 \\
\hline $\mathrm{O} 25 \cdots \mathrm{C} 33$ & $3.356(7)$ & $\mathrm{H} 9 \cdots \mathrm{O} 21^{\mathrm{vi}}$ & 2.8300 \\
\hline $\mathrm{O} 25 \cdots \mathrm{N} 27$ & $2.813(6)$ & $\mathrm{H} 9 \cdots \mathrm{C} 20^{\mathrm{vi}}$ & 2.5700 \\
\hline $\mathrm{O} 25 \cdots \mathrm{O} 1^{\mathrm{x}}$ & 2.7633 & $\mathrm{H} 9 \cdots \mathrm{O} 22^{\mathrm{vi}}$ & 1.7400 \\
\hline $\mathrm{O} 1 \cdots \mathrm{H} 51$ & 1.9700 & $\mathrm{H} 34 \cdots \mathrm{H} 48$ & $2.430(18)$ \\
\hline 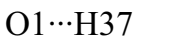 & 2.5800 & $\mathrm{H} 34 \cdots \mathrm{O} 17$ & $2.793(7)$ \\
\hline $\mathrm{O} 4 \cdots \mathrm{H} 52^{\mathrm{vii}}$ & 2.7900 & $\mathrm{H} 34 \cdots \mathrm{C} 28^{\text {vii }}$ & $3.034(8)$ \\
\hline $\mathrm{O} 4 \cdots \mathrm{H} 38^{\text {iv }}$ & 1.8600 & $\mathrm{H} 34 \cdots \mathrm{C} 29^{\mathrm{vii}}$ & $3.041(8)$ \\
\hline $\mathrm{O} 4 \cdots \mathrm{H} 3$ & 2.0200 & $\mathrm{H} 35 \cdots \mathrm{C} 18$ & $3.039(8)$ \\
\hline 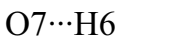 & 1.8200 & $\mathrm{H} 35 \cdots \mathrm{S} 10^{\mathrm{iii}}$ & $2.869(6)$ \\
\hline $\mathrm{O} 7 \cdots \mathrm{H}^{\mathrm{v}}$ & 1.8000 & $\mathrm{H} 35 \cdots \mathrm{H} 50^{\mathrm{x}}$ & $2.537(16)$ \\
\hline $\mathrm{O} 17 \cdots \mathrm{H} 8$ & 1.8400 & $\mathrm{H} 36 \cdots \mathrm{H} 2$ & 2.5000 \\
\hline $\mathrm{O} 17 \cdots \mathrm{H} 42^{\mathrm{vii}}$ & $2.831(18)$ & $\mathrm{H} 36 \cdots \mathrm{O} 25$ & $2.501(9)$ \\
\hline $\mathrm{O} 17 \cdots \mathrm{H} 34$ & $2.793(7)$ & 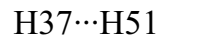 & $2.14(4)$ \\
\hline $\mathrm{O} 17 \cdots \mathrm{H} 43^{\mathrm{viii}}$ & $2.384(16)$ & $\mathrm{H} 37 \cdots \mathrm{O} 1$ & 2.5800 \\
\hline $\mathrm{O} 21 \cdots \mathrm{H} 44^{\mathrm{viii}}$ & $2.78(2)$ & $\mathrm{H} 37 \cdots \mathrm{H} 41$ & $2.29(2)$ \\
\hline $\mathrm{O} 21 \cdots \mathrm{H} 39^{\text {viii }}$ & $1.83(3)$ & $\mathrm{H} 37 \cdots \mathrm{O} 22^{\mathrm{xi}}$ & $2.858(9)$ \\
\hline $\mathrm{O} 21 \cdots \mathrm{H} 9^{\text {iх }}$ & 2.8300 & $\mathrm{H} 38 \cdots \mathrm{H} 52^{\mathrm{xiii}}$ & $2.54(3)$ \\
\hline $\mathrm{O} 21 \cdots \mathrm{H} 47$ & $2.87(3)$ & $\mathrm{H} 38 \cdots \mathrm{H} 5^{\mathrm{v}}$ & 2.5000 \\
\hline $\mathrm{O} 22 \cdots \mathrm{H} 37^{\mathrm{vii}}$ & $2.858(9)$ & $\mathrm{H} 38{ }^{\cdots} \cdot \mathrm{H} 6^{v}$ & 2.2600 \\
\hline $\mathrm{O} 22 \cdots \mathrm{H} 40^{\mathrm{vii}}$ & $1.80(2)$ & $\mathrm{H} 38 \cdots \mathrm{O} 4^{v}$ & 1.8600 \\
\hline $\mathrm{O} 22 \cdots \mathrm{H} 9^{\mathrm{ix}}$ & 1.7400 & $\mathrm{H} 39 \cdots \mathrm{O} 25$ & $2.59(3)$ \\
\hline $\mathrm{O} 22 \cdots \mathrm{H} 49$ & 2.704 (13) & H $39 \cdots \mathrm{C} 33$ & $2.790(19)$ \\
\hline $\mathrm{O} 25 \cdots \mathrm{H} 36$ & $2.501(9)$ & 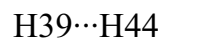 & $2.29(3)$ \\
\hline $\mathrm{O} 25 \cdots \mathrm{H} 39$ & $2.59(3)$ & $\mathrm{H} 39 \cdots \mathrm{O} 21^{\mathrm{xii}}$ & $1.83(3)$ \\
\hline $\mathrm{O} 25 \cdots \mathrm{H} 41^{\mathrm{x}}$ & $2.617(17)$ & $\mathrm{H} 39 \cdots \mathrm{C} 20^{\mathrm{xii}}$ & $2.94(3)$ \\
\hline
\end{tabular}




\begin{tabular}{|c|c|}
\hline $\mathrm{O} 25 \cdots \mathrm{H} 44$ & $2.89(2)$ \\
\hline $\mathrm{O} 25 \cdots \mathrm{H} 2$ & 1.7800 \\
\hline $\mathrm{N} 13 \cdots \mathrm{S} 10$ & $2.602(4)$ \\
\hline $\mathrm{N} 13 \cdots \mathrm{O} 21$ & $2.715(5)$ \\
\hline $\mathrm{N} 13 \cdots \mathrm{N} 23$ & $3.267(5)$ \\
\hline $\mathrm{N} 23 \cdots \mathrm{O} 17$ & $3.142(5)$ \\
\hline $\mathrm{N} 23 \cdots \mathrm{O} 1$ & 2.9161 \\
\hline $\mathrm{N} 23 \cdots \mathrm{S} 10$ & $3.149(5)$ \\
\hline $\mathrm{N} 23 \cdots \mathrm{N} 13$ & $3.267(5)$ \\
\hline $\mathrm{N} 27 \cdots \mathrm{O} 4^{\mathrm{v}}$ & 2.8272 \\
\hline $\mathrm{N} 27 \cdots \mathrm{O} 22^{\mathrm{xi}}$ & $2.688(6)$ \\
\hline $\mathrm{N} 27 \cdots \mathrm{O} 25$ & $2.813(6)$ \\
\hline $\mathrm{N} 27 \cdots \mathrm{O} 21^{\mathrm{xii}}$ & $2.742(6)$ \\
\hline 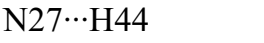 & $2.87(2)$ \\
\hline $\mathrm{C} 18 \cdots \mathrm{O} 22$ & $3.213(7)$ \\
\hline $\mathrm{C} 19 \cdots \mathrm{O} 22$ & $3.305(6)$ \\
\hline $\mathrm{C} 26 \cdots \mathrm{O} 22^{\mathrm{xi}}$ & $3.109(5)$ \\
\hline $\mathrm{C} 28 \cdots \mathrm{O} 22^{\mathrm{xi}}$ & $3.356(6)$ \\
\hline $\mathrm{C} 30 \cdots \mathrm{O} 17^{\mathrm{xi}}$ & $3.067(7)$ \\
\hline $\mathrm{C} 31 \cdots \mathrm{O} 17^{\mathrm{xi}}$ & $3.156(7)$ \\
\hline $\mathrm{C} 32 \cdots \mathrm{O} 17^{\mathrm{xii}}$ & $3.228(7)$ \\
\hline $\mathrm{C} 33 \cdots \mathrm{O} 25$ & $3.356(7)$ \\
\hline $\mathrm{C} 14 \cdots \mathrm{H} 47$ & $3.09(2)$ \\
\hline $\mathrm{C} 16 \cdots \mathrm{H} 43^{\text {viii }}$ & $3.07(2)$ \\
\hline $\mathrm{C} 16 \cdots \mathrm{H} 8$ & 2.8000 \\
\hline $\mathrm{C} 18 \cdots \mathrm{H} 42^{\mathrm{iii}}$ & $2.803(17)$ \\
\hline 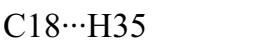 & $3.039(8)$ \\
\hline $\mathrm{C} 20 \cdots \mathrm{H} 47$ & $2.50(3)$ \\
\hline $\mathrm{C} 20 \cdots \mathrm{H} 9^{\text {ix }}$ & 2.5700 \\
\hline $\mathrm{C} 20 \cdots \mathrm{H} 39^{\text {viii }}$ & $2.94(3)$ \\
\hline $\mathrm{C} 20 \cdots \mathrm{H} 40^{\text {vii }}$ & $2.90(2)$ \\
\hline $\mathrm{C} 24 \cdots \mathrm{H} 2$ & 2.7700 \\
\hline $\mathrm{C} 24 \cdots \mathrm{H} 44$ & $3.06(2)$ \\
\hline $\mathrm{C} 28 \cdots \mathrm{H} 34^{\mathrm{xi}}$ & $3.034(8)$ \\
\hline $\mathrm{C} 29 \cdots \mathrm{H} 34^{\mathrm{xi}}$ & $3.041(8)$ \\
\hline $\mathrm{C} 30 \cdots \mathrm{H} 47^{\mathrm{ii}}$ & $2.77(2)$ \\
\hline $\mathrm{C} 32 \cdots \mathrm{H} 46^{\mathrm{iii}}$ & $2.93(3)$ \\
\hline 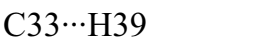 & $2.790(19)$ \\
\hline $\mathrm{C} 33 \cdots \mathrm{H} 46^{\mathrm{iii}}$ & $2.92(2)$ \\
\hline $\mathrm{C} 11-\mathrm{S} 10-\mathrm{C} 14$ & $90.10(18)$ \\
\hline 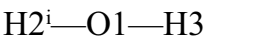 & 113.00 \\
\hline $\mathrm{H} 5-\mathrm{O} 4-\mathrm{H} 6$ & 128.00 \\
\hline $\mathrm{H} 8-\mathrm{O} 7-\mathrm{H} 9$ & 103.00 \\
\hline $\mathrm{C} 12-\mathrm{N} 13-\mathrm{C} 14$ & $117.0(3)$ \\
\hline $\mathrm{C} 12-\mathrm{N} 13-\mathrm{C} 16$ & $128.1(3)$ \\
\hline C14-N13-C16 & $93.1(3)$ \\
\hline $\mathrm{C} 15-\mathrm{N} 23-\mathrm{C} 24$ & $123.3(3)$ \\
\hline
\end{tabular}

$\mathrm{H} 40 \cdots \mathrm{H} 44^{\mathrm{xi}}$

$\mathrm{H} 40 \cdots \mathrm{O} 22^{\mathrm{xi}}$

$\mathrm{H} 40 \cdots \mathrm{C} 20^{\mathrm{xi}}$

$\mathrm{H} 41 \cdots \mathrm{O} 25^{\mathrm{i}}$

$\mathrm{H} 41 \cdots \mathrm{H} 37$

$\mathrm{H} 42 \cdots \mathrm{C} 18^{\mathrm{ii}}$

$\mathrm{H} 42 \cdots \mathrm{O} 17^{\mathrm{xi}}$

$\mathrm{H} 42 \cdots \mathrm{H} 47^{\mathrm{ii}}$

$\mathrm{H} 43 \cdots \mathrm{C} 16^{\mathrm{xii}}$

$\mathrm{H} 43 \cdots \mathrm{H} 8^{\mathrm{xii}}$

$\mathrm{H} 43 \cdots \mathrm{O} 17^{\mathrm{xii}}$

$\mathrm{H} 44 \cdots \mathrm{C} 24$

$\mathrm{H} 44 \cdots \mathrm{O} 25$

$\mathrm{H} 44 \cdots \mathrm{N} 27$

$\mathrm{H} 44 \cdots \mathrm{O} 21^{\mathrm{xii}}$

$\mathrm{H} 44 \cdots \mathrm{H} 39$

$\mathrm{H} 46{ }^{\cdots} \mathrm{C} 32^{\mathrm{ii}}$

$\mathrm{H} 46 \cdot \cdots \mathrm{H} 49$

$\mathrm{H} 46{ }^{\cdots} \mathrm{C} 33^{\mathrm{ii}}$

$\mathrm{H} 47 \cdots \mathrm{C} 14$

$\mathrm{H} 47 \cdots \mathrm{O} 21$

$\mathrm{H} 47 \cdots \mathrm{H} 42^{\mathrm{iii}}$

$\mathrm{H} 47 \cdots \mathrm{C} 30^{\mathrm{iii}}$

$\mathrm{H} 47 \cdots \mathrm{C} 20$

$\mathrm{H} 48 \cdots \mathrm{H} 34$

$\mathrm{H} 49 \cdots \mathrm{H} 40^{\mathrm{vii}}$

$\mathrm{H} 49 \cdots \mathrm{H} 46$

$\mathrm{H} 49 \cdots \mathrm{O} 22$

$\mathrm{H} 50 \cdots \mathrm{S} 10^{\mathrm{ii}}$

$\mathrm{H} 50 \cdots \mathrm{H} 35^{\mathrm{i}}$

$\mathrm{H} 51 \cdots \mathrm{S} 10$

$\mathrm{H} 51 \cdots \mathrm{O} 1$

$\mathrm{H} 51 \cdots \mathrm{H}^{\mathrm{i}}$

$\mathrm{H} 51 \cdots \mathrm{H} 3$

$\mathrm{H} 51 \cdots \mathrm{H} 37$

$\mathrm{H} 52 \cdots \mathrm{O} 4^{\mathrm{xi}}$

$\mathrm{H} 52 \cdots \mathrm{H} 6^{\mathrm{xi}}$

$\mathrm{H} 52 \cdots \mathrm{H} 38^{\mathrm{xiv}}$

$\mathrm{C} 26-\mathrm{C} 28-\mathrm{C} 33$

$\mathrm{C} 29-\mathrm{C} 28-\mathrm{C} 33$

$\mathrm{C} 26-\mathrm{C} 28-\mathrm{C} 29$

$\mathrm{C} 28-\mathrm{C} 29-\mathrm{C} 30$

$\mathrm{C} 29-\mathrm{C} 30-\mathrm{C} 31$

$\mathrm{C} 30-\mathrm{C} 31-\mathrm{C} 32$

C $31-\mathrm{C} 32-\mathrm{C} 33$

$\mathrm{C} 28-\mathrm{C} 33-\mathrm{C} 32$
$2.30(4)$

1.80 (2)

$2.90(2)$

2.617 (17)

$2.29(2)$

2.803 (17)

2.831 (18)

2.05 (3)

3.07 (2)

2.4800

2.384 (16)

$3.06(2)$

2.89 (2)

$2.87(2)$

2.78 (2)

$2.29(3)$

2.93 (3)

$2.26(3)$

$2.92(2)$

3.09 (2)

2.87 (3)

2.05 (3)

2.77 (2)

2.50 (3)

2.430 (18)

2.30 (4)

$2.26(3)$

2.704 (13)

$3.16(3)$

2.537 (16)

2.83 (4)

1.9700

2.5800

2.4700

2.14 (4)

2.7900

2.5600

2.54 (3)

$120.6(4)$

119.5 (5)

119.5 (4)

119.8 (5)

120.1 (5)

119.5 (5)

119.9 (5)

120.0 (4) 


\begin{tabular}{|c|c|}
\hline $\mathrm{C} 24-\mathrm{N} 23-\mathrm{H} 51$ & $118(2)$ \\
\hline $\mathrm{C} 15-\mathrm{N} 23-\mathrm{H} 51$ & $119(2)$ \\
\hline $\mathrm{H} 38-\mathrm{N} 27-\mathrm{H} 40$ & $109(2)$ \\
\hline H38-N27-H39 & $109(3)$ \\
\hline $\mathrm{C} 26-\mathrm{N} 27-\mathrm{H} 38$ & $109.7(11)$ \\
\hline $\mathrm{C} 26-\mathrm{N} 27-\mathrm{H} 40$ & $109.7(14)$ \\
\hline $\mathrm{H} 39-\mathrm{N} 27-\mathrm{H} 40$ & $110(2)$ \\
\hline $\mathrm{C} 26-\mathrm{N} 27-\mathrm{H} 39$ & $109.5(9)$ \\
\hline $\mathrm{S} 10-\mathrm{C} 11-\mathrm{C} 19$ & 107.5 \\
\hline $\mathrm{C} 12-\mathrm{C} 11-\mathrm{C} 18$ & $111.9(3)$ \\
\hline $\mathrm{S} 10-\mathrm{C} 11-\mathrm{C} 12$ & $104.6(2)$ \\
\hline $\mathrm{C} 12-\mathrm{C} 11-\mathrm{C} 19$ & $112.9(3)$ \\
\hline $\mathrm{C} 18-\mathrm{C} 11-\mathrm{C} 19$ & $109.7(3)$ \\
\hline $\mathrm{S} 10-\mathrm{C} 11-\mathrm{C} 18$ & $110.0(3)$ \\
\hline $\mathrm{C} 11-\mathrm{C} 12-\mathrm{C} 20$ & $114.3(3)$ \\
\hline $\mathrm{N} 13-\mathrm{C} 12-\mathrm{C} 11$ & $105.6(3)$ \\
\hline $\mathrm{N} 13-\mathrm{C} 12-\mathrm{C} 20$ & $112.8(3)$ \\
\hline $\mathrm{N} 13-\mathrm{C} 14-\mathrm{C} 15$ & $87.1(2)$ \\
\hline $\mathrm{S} 10-\mathrm{C} 14-\mathrm{N} 13$ & $103.6(2)$ \\
\hline $\mathrm{S} 10-\mathrm{C} 14-\mathrm{C} 15$ & $119.5(2)$ \\
\hline $\mathrm{N} 23-\mathrm{C} 15-\mathrm{C} 16$ & 115.5 \\
\hline $\mathrm{C} 14-\mathrm{C} 15-\mathrm{C} 16$ & $85.1(3)$ \\
\hline $\mathrm{N} 23-\mathrm{C} 15-\mathrm{C} 14$ & $116.5(3)$ \\
\hline $\mathrm{O} 17-\mathrm{C} 16-\mathrm{C} 15$ & $135.4(3)$ \\
\hline $\mathrm{O} 17-\mathrm{C} 16-\mathrm{N} 13$ & $131.0(4)$ \\
\hline $\mathrm{N} 13-\mathrm{C} 16-\mathrm{C} 15$ & $93.0(3)$ \\
\hline $\mathrm{O} 21-\mathrm{C} 20-\mathrm{C} 12$ & $118.0(4)$ \\
\hline $\mathrm{O} 21-\mathrm{C} 20-\mathrm{O} 22$ & $126.3(4)$ \\
\hline $\mathrm{O} 22-\mathrm{C} 20-\mathrm{C} 12$ & $115.6(4)$ \\
\hline $\mathrm{O} 25-\mathrm{C} 24-\mathrm{N} 23$ & $124.5(5)$ \\
\hline $\mathrm{O} 25-\mathrm{C} 24-\mathrm{C} 26$ & $122.1(5)$ \\
\hline $\mathrm{N} 23-\mathrm{C} 24-\mathrm{C} 26$ & $113.2(3)$ \\
\hline $\mathrm{C} 24-\mathrm{C} 26-\mathrm{C} 28$ & $110.1(3)$ \\
\hline $\mathrm{N} 27-\mathrm{C} 26-\mathrm{C} 24$ & $110.0(3)$ \\
\hline $\mathrm{N} 27-\mathrm{C} 26-\mathrm{C} 28$ & $112.9(3)$ \\
\hline $\mathrm{C} 14-\mathrm{S} 10-\mathrm{C} 11-\mathrm{C} 12$ & $-39.8(2)$ \\
\hline $\mathrm{C} 14-\mathrm{S} 10-\mathrm{C} 11-\mathrm{C} 18$ & $80.5(3)$ \\
\hline $\mathrm{C} 14-\mathrm{S} 10-\mathrm{C} 11-\mathrm{C} 19$ & $-160.1(3)$ \\
\hline $\mathrm{C} 11-\mathrm{S} 10-\mathrm{C} 14-\mathrm{N} 13$ & $37.1(2)$ \\
\hline $\mathrm{C} 11-\mathrm{S} 10-\mathrm{C} 14-\mathrm{C} 15$ & $131.6(3)$ \\
\hline $\mathrm{C} 14-\mathrm{N} 13-\mathrm{C} 16-\mathrm{C} 15$ & $10.2(3)$ \\
\hline $\mathrm{C} 16-\mathrm{N} 13-\mathrm{C} 14-\mathrm{C} 15$ & $-10.0(3)$ \\
\hline $\mathrm{C} 14-\mathrm{N} 13-\mathrm{C} 12-\mathrm{C} 11$ & $-2.6(4)$ \\
\hline $\mathrm{C} 12-\mathrm{N} 13-\mathrm{C} 14-\mathrm{C} 15$ & $-146.1(3)$ \\
\hline $\mathrm{C} 14-\mathrm{N} 13-\mathrm{C} 12-\mathrm{C} 20$ & $-128.1(4)$ \\
\hline $\mathrm{C} 16-\mathrm{N} 13-\mathrm{C} 12-\mathrm{C} 20$ & $113.4(5)$ \\
\hline $\mathrm{C} 16-\mathrm{N} 13-\mathrm{C} 12-\mathrm{C} 11$ & $-121.1(4)$ \\
\hline
\end{tabular}

\begin{tabular}{|c|c|}
\hline $\mathrm{N} 13-\mathrm{C} 12-\mathrm{H} 34$ & $103.8(4)$ \\
\hline $\mathrm{C} 11-\mathrm{C} 12-\mathrm{H} 34$ & $112.0(5)$ \\
\hline $\mathrm{C} 20-\mathrm{C} 12-\mathrm{H} 34$ & $107.8(5)$ \\
\hline $\mathrm{C} 15-\mathrm{C} 14-\mathrm{H} 35$ & $118.7(4)$ \\
\hline $\mathrm{N} 13-\mathrm{C} 14-\mathrm{H} 35$ & $116.8(5)$ \\
\hline $\mathrm{S} 10-\mathrm{C} 14-\mathrm{H} 35$ & $108.6(4)$ \\
\hline $\mathrm{C} 16-\mathrm{C} 15-\mathrm{H} 36$ & $115.8(5)$ \\
\hline $\mathrm{C} 14-\mathrm{C} 15-\mathrm{H} 36$ & $108.0(5)$ \\
\hline $\mathrm{N} 23-\mathrm{C} 15-\mathrm{H} 36$ & $112.9(5)$ \\
\hline $\mathrm{C} 11-\mathrm{C} 18-\mathrm{H} 45$ & $109.4(11)$ \\
\hline $\mathrm{C} 11-\mathrm{C} 18-\mathrm{H} 46$ & $109.2(11)$ \\
\hline $\mathrm{C} 11-\mathrm{C} 18-\mathrm{H} 47$ & $109.8(6)$ \\
\hline $\mathrm{H} 46-\mathrm{C} 18-\mathrm{H} 47$ & $110(2)$ \\
\hline $\mathrm{H} 45-\mathrm{C} 18-\mathrm{H} 46$ & $109(2)$ \\
\hline $\mathrm{H} 45-\mathrm{C} 18-\mathrm{H} 47$ & $110(3)$ \\
\hline $\mathrm{C} 11-\mathrm{C} 19-\mathrm{H} 48$ & $109.4(8)$ \\
\hline $\mathrm{C} 11-\mathrm{C} 19-\mathrm{H} 49$ & $109.4(6)$ \\
\hline $\mathrm{C} 11-\mathrm{C} 19-\mathrm{H} 50$ & $109.3(9)$ \\
\hline $\mathrm{H} 48-\mathrm{C} 19-\mathrm{H} 49$ & $110(2)$ \\
\hline $\mathrm{H} 49-\mathrm{C} 19-\mathrm{H} 50$ & $109.2(19)$ \\
\hline $\mathrm{H} 48-\mathrm{C} 19-\mathrm{H} 50$ & $109(2)$ \\
\hline $\mathrm{C} 28-\mathrm{C} 26-\mathrm{H} 37$ & $106.8(5)$ \\
\hline $\mathrm{C} 24-\mathrm{C} 26-\mathrm{H} 37$ & $109.0(5)$ \\
\hline $\mathrm{N} 27-\mathrm{C} 26-\mathrm{H} 37$ & $107.9(5)$ \\
\hline $\mathrm{C} 28-\mathrm{C} 29-\mathrm{H} 41$ & $120.0(12)$ \\
\hline $\mathrm{C} 30-\mathrm{C} 29-\mathrm{H} 41$ & $120.0(11)$ \\
\hline $\mathrm{C} 29-\mathrm{C} 30-\mathrm{H} 42$ & $119.4(11)$ \\
\hline $\mathrm{C} 31-\mathrm{C} 30-\mathrm{H} 42$ & $119.6(11)$ \\
\hline $\mathrm{C} 32-\mathrm{C} 31-\mathrm{H} 52$ & $120.0(9)$ \\
\hline $\mathrm{C} 30-\mathrm{C} 31-\mathrm{H} 52$ & $120.3(9)$ \\
\hline $\mathrm{C} 31-\mathrm{C} 32-\mathrm{H} 43$ & $120.1(11)$ \\
\hline $\mathrm{C} 33-\mathrm{C} 32-\mathrm{H} 43$ & $120.1(11)$ \\
\hline $\mathrm{C} 28-\mathrm{C} 33-\mathrm{H} 44$ & $119.9(12)$ \\
\hline $\mathrm{C} 32-\mathrm{C} 33-\mathrm{H} 44$ & $119.9(12)$ \\
\hline $\mathrm{C} 11-\mathrm{C} 12-\mathrm{C} 20-\mathrm{O} 21$ & $-124.3(5$ \\
\hline $\mathrm{C} 11-\mathrm{C} 12-\mathrm{C} 20-\mathrm{O} 22$ & $59.0(6)$ \\
\hline $\mathrm{N} 13-\mathrm{C} 12-\mathrm{C} 20-\mathrm{O} 21$ & $-3.6(6)$ \\
\hline $\mathrm{S} 10-\mathrm{C} 14-\mathrm{C} 15-\mathrm{C} 16$ & $-95.1(3)$ \\
\hline $\mathrm{S} 10-\mathrm{C} 14-\mathrm{C} 15-\mathrm{N} 23$ & $21.1(4)$ \\
\hline $\mathrm{N} 13-\mathrm{C} 14-\mathrm{C} 15-\mathrm{C} 16$ & $9.0(3)$ \\
\hline $\mathrm{N} 13-\mathrm{C} 14-\mathrm{C} 15-\mathrm{N} 23$ & $125.1(3)$ \\
\hline $\mathrm{N} 23-\mathrm{C} 15-\mathrm{C} 16-\mathrm{O} 17$ & $44.6(8)$ \\
\hline $\mathrm{C} 14-\mathrm{C} 15-\mathrm{C} 16-\mathrm{O} 17$ & $161.7(7)$ \\
\hline $\mathrm{C} 14-\mathrm{C} 15-\mathrm{C} 16-\mathrm{N} 13$ & $-9.8(3)$ \\
\hline $\mathrm{N} 23-\mathrm{C} 15-\mathrm{C} 16-\mathrm{N} 13$ & $-126.9(3$ \\
\hline $\mathrm{N} 23-\mathrm{C} 24-\mathrm{C} 26-\mathrm{C} 28$ & $89.1(5)$ \\
\hline
\end{tabular}




$\begin{array}{llll}\mathrm{C} 12-\mathrm{N} 13-\mathrm{C} 14-\mathrm{S} 10 & -26.5(3) & \mathrm{O} 25-\mathrm{C} 24-\mathrm{C} 26-\mathrm{N} 27 & 37.9(7) \\ \mathrm{C} 16-\mathrm{N} 13-\mathrm{C} 14-\mathrm{S} 10 & 109.7(3) & \mathrm{O} 25-\mathrm{C} 24-\mathrm{C} 26-\mathrm{C} 28 & -87.2(6) \\ \mathrm{C} 12-\mathrm{N} 13-\mathrm{C} 16-\mathrm{O} 17 & -33.5(8) & \mathrm{N} 23-\mathrm{C} 24-\mathrm{C} 26-\mathrm{N} 27 & -145.8(4) \\ \mathrm{C} 12-\mathrm{N} 13-\mathrm{C} 16-\mathrm{C} 15 & 138.6(4) & \mathrm{N} 27-\mathrm{C} 26-\mathrm{C} 28-\mathrm{C} 29 & 132.8(5) \\ \mathrm{C} 14-\mathrm{N} 13-\mathrm{C} 16-\mathrm{O} 17 & -161.9(6) & \mathrm{N} 27-\mathrm{C} 26-\mathrm{C} 28-\mathrm{C} 33 & -54.6(6) \\ \mathrm{C} 15-\mathrm{N} 23-\mathrm{C} 24-\mathrm{O} 25 & 0.3(9) & \mathrm{C} 24-\mathrm{C} 26-\mathrm{C} 28-\mathrm{C} 33 & 68.7(6) \\ \mathrm{C} 15-\mathrm{N} 23-\mathrm{C} 24-\mathrm{C} 26 & -175.9(4) & \mathrm{C} 24-\mathrm{C} 26-\mathrm{C} 28-\mathrm{C} 29 & -103.8(5) \\ \mathrm{C} 24-\mathrm{N} 23-\mathrm{C} 15-\mathrm{C} 14 & 136.1(5) & \mathrm{C} 26-\mathrm{C} 28-\mathrm{C} 33-\mathrm{C} 32 & -174.8(5) \\ \mathrm{C} 24-\mathrm{N} 23-\mathrm{C} 15-\mathrm{C} 16 & -126.3(5) & \mathrm{C} 26-\mathrm{C} 28-\mathrm{C} 29-\mathrm{C} 30 & 172.0(5) \\ \mathrm{S} 10-\mathrm{C} 11-\mathrm{C} 12-\mathrm{C} 20 & 154.2(3) & \mathrm{C} 33-\mathrm{C} 28-\mathrm{C} 29-\mathrm{C} 30 & -0.6(8) \\ \mathrm{S} 10-\mathrm{C} 11-\mathrm{C} 12-\mathrm{N} 13 & 29.6(3) & \mathrm{C} 29-\mathrm{C} 28-\mathrm{C} 33-\mathrm{C} 32 & -2.2(8) \\ \mathrm{C} 19-\mathrm{C} 11-\mathrm{C} 12-\mathrm{N} 13 & 146.2(3) & \mathrm{C} 28-\mathrm{C} 29-\mathrm{C} 30-\mathrm{C} 31 & 8.5(8) \\ \mathrm{C} 19-\mathrm{C} 11-\mathrm{C} 12-\mathrm{C} 20 & -89.2(4) & \mathrm{C} 29-\mathrm{C} 30-\mathrm{C} 31-\mathrm{C} 32 & -13.5(8) \\ \mathrm{C} 18-\mathrm{C} 11-\mathrm{C} 12-\mathrm{C} 20 & 35.1(4) & \mathrm{C} 30-\mathrm{C} 31-\mathrm{C} 32-\mathrm{C} 33 & 10.6(9) \\ \mathrm{C} 18-\mathrm{C} 11-\mathrm{C} 12-\mathrm{N} 13 & -89.5(4) & \mathrm{C} 31-\mathrm{C} 32-\mathrm{C} 33-\mathrm{C} 28 & -2.8(9) \\ \mathrm{N} 13-\mathrm{C} 12-\mathrm{C} 20-\mathrm{O} 22 & 179.7(4) & & \end{array}$

Symmetry codes: (i) $x, y, z-1$; (ii) $-x+1 / 2,-y+1, z-1 / 2$; (iii) $-x+1 / 2,-y+1, z+1 / 2$; (iv) $-x+3 / 2,-y+1, z-1 / 2$; (v) $-x+3 / 2,-y+1, z+1 / 2$; (vi) $x+1 / 2,-y+1 / 2$, $-z+1$; (vii) $-x+1, y-1 / 2,-z+1 / 2$; (viii) $-x+1, y-1 / 2,-z+3 / 2$; (ix) $x-1 / 2,-y+1 / 2,-z+1$; (x) $x, y, z+1$; (xi) $-x+1, y+1 / 2,-z+1 / 2$; (xii) $-x+1, y+1 / 2,-z+3 / 2$; (xiii) $x+1 / 2,-y+3 / 2,-z+1$; (xiv) $x-1 / 2,-y+3 / 2,-z+1$.

Hydrogen-bond geometry $\left(\AA,{ }^{\circ}\right)$

\begin{tabular}{lllll}
\hline$D-\mathrm{H}^{\prime} \cdots A$ & $D-\mathrm{H}$ & $\mathrm{H} \cdots A$ & $D \cdots A$ & $D-\mathrm{H} \cdots A$ \\
\hline $\mathrm{O} 1-\mathrm{H} 3 \cdots \mathrm{O} 4$ & 0.98 & 2.02 & 2.9968 & 180 \\
$\mathrm{O} 4-\mathrm{H} 5 \cdots \mathrm{O} 7^{\text {iv }}$ & 0.98 & 1.80 & 2.7850 & 180 \\
$\mathrm{O} 4-\mathrm{H} 6 \cdots \mathrm{O} 7$ & 0.98 & 1.82 & 2.7967 & 180 \\
$\mathrm{O} 7-\mathrm{H} 8 \cdots \mathrm{O} 17$ & 0.98 & 1.84 & 2.8225 & 180 \\
$\mathrm{O} 7-\mathrm{H} 9 \cdots \mathrm{O} 22^{\mathrm{vi}}$ & 0.98 & 1.74 & 2.7166 & 179 \\
$\mathrm{~N} 27-\mathrm{H} 38 \cdots \mathrm{O} 4^{\mathrm{v}}$ & $1.001(15)$ & 1.86 & 2.8272 & 161 \\
$\mathrm{~N} 27-\mathrm{H} 39 \cdots \mathrm{O} 21^{\mathrm{xii}}$ & $1.00(3)$ & $1.83(3)$ & $2.742(6)$ & $151.1(15)$ \\
$\mathrm{N} 27-\mathrm{H} 40 \cdots \mathrm{O} 22^{\mathrm{xi}}$ & $1.00(3)$ & $1.80(2)$ & $2.688(6)$ & $147(2)$ \\
$\mathrm{N} 23-\mathrm{H} 51 \cdots \mathrm{O} 1$ & $1.002(10)$ & 1.97 & 2.9161 & 156 \\
$\mathrm{C} 14-\mathrm{H} 35 \cdots \mathrm{S} 10^{\mathrm{iii}}$ & $0.999(6)$ & $2.869(6)$ & $3.815(4)$ & $158.3(6)$ \\
$\mathrm{C} 26-\mathrm{H} 37 \cdots \mathrm{O} 1$ & $1.080(7)$ & 2.58 & 3.5092 & 143 \\
$\mathrm{C} 32-\mathrm{H} 43 \cdots \mathrm{O} 17^{\mathrm{xii}}$ & $1.079(19)$ & $2.384(16)$ & $3.228(7)$ & $134.0(16)$ \\
\end{tabular}

Symmetry codes: (iii) $-x+1 / 2,-y+1, z+1 / 2$; (iv) $-x+3 / 2,-y+1, z-1 / 2$; (v) $-x+3 / 2,-y+1, z+1 / 2$; (vi) $x+1 / 2,-y+1 / 2,-z+1$; (xi) $-x+1, y+1 / 2,-z+1 / 2$; (xii) $-x+1, y+1 / 2,-z+3 / 2$. 